\title{
Vulnerabilidade ao estresse e alimentação: um estudo no contexto do trabalho
}

\author{
Stress vulnerability and feeding: a study in the work context \\ Francieli Dalla Costa ${ }^{1}$, Carla Rosane Paz Arruda Teo ${ }^{2} \bowtie$, Josiane Schadeck de Almeida ${ }^{3}$ \\ ' Serviço Nacional de Aprendizagem Industrial (SENAI), Unidade São Lourenço d'Oeste, SC, Brasil. \\ 2 Programa de Pós-Graduação em Ciências da Saúde da Universidade Comunitária da Região de Chapecó (Unochapecó). Chapecó, SC, Brasil. \\ 3 Área de Ciências da Saúde da Universidade Comunitária da Região de Chapecó (Unochapecó). Chapecó, SC, Brasil.
}

Fonte de recursos: Estudo conduzido com recursos da Taxa de Apoio à Produção Científica do Programa de Pós-Graduação em Ciências da Saúde da Universidade Comunitária da Região de Chapecó.

Artigo elaborado a partir da dissertação de Francieli Dalla Costa, "Relações entre vulnerabilidade ao estresse e alimentação no contexto do trabalho", defendida em agosto de 2014.

\section{RESUMO}

Objetivos: Analisar a vulnerabilidade ao estresse no contexto do trabalho e suas implicações com a alimentação.

Métodos: Estudo transversal com trabalhadores de uma indústria do estado de Santa Catarina. Os instrumentos utilizados foram a Escala de Vulnerabilidade ao Estresse no Trabalho (EVENT) e um questionário estruturado. Foram analisadas seis variáveis sociodemográficas e ocupacionais (idade, sexo, escolaridade, turno de trabalho, setor/unidade de trabalho, tempo de trabalho), nível de vulnerabilidade ao estresse (segundo a EVENT) e, ainda, cinco variáveis alimentares onde o nível de estresse considerado foi o autorreferido e não a vulnerabilidade avaliada pela EVENT. A análise de dados empregou o teste t de Student e o qui-quadrado de Pearson e de tendência linear, sendo adotado o nível de significância de $5 \%(\mathrm{p} \leq 0,05)$.

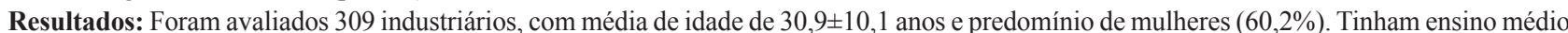
completo $44,7 \%$ e ensino fundamental completo $25,5 \%$. O tempo de trabalho na empresa teve mediana de 23 meses (intervalo interquartil 7-57); $81,6 \%$ trabalhavam no período diurno e $18,4 \%$ no noturno. O escore médio de vulnerabilidade ao estresse foi $22 \pm 11,6$ pontos; 249 ( $80,6 \%$ ) participantes foram classificados nos níveis inferiores de vulnerabilidade ao estresse e $60(19,4 \%)$ nos superiores. Houve associação entre elevada vulnerabilidade ao estresse pela EVENT e alteração autorreferida na alimentação em condições de estresse $(\mathrm{p}=0,028)$. A prevalência de elevada vulnerabilidade ao estresse aumentou com o tempo de empresa $(\mathrm{p}=0,034)$. Sob estresse autorreferido, as mulheres relataram ter mais fome, alteração na alimentação e preferência por doces $(\mathrm{p}<0,001)$.

Conclusões: $\mathrm{O}$ grupo de trabalhadores na indústria apresentou prevalência de níveis inferiores de vulnerabilidade ao estresse, com a pressão no trabalho representando o principal fator estressor. Níveis superiores de vulnerabilidade ao estresse estiveram associados ao aumento do tempo de trabalho na empresa, assim como à percepção de que o trabalho causa estresse e interfere na alimentação. A constatação de que, sob condição de estresse autorreferido relacionado ao trabalho, a prevalência de alteração percebida na alimentação foi maior entre as mulheres, associada ao aumento da fome e à preferência por doces, indica uma maior suscetibilidade deste grupo a essas alterações, com potenciais riscos de agravos à saúde.

DESCRITORES: comportamento alimentar; esgotamento profissional; saúde do trabalhador; vulnerabilidade em saúde; estresse psicológico.

\section{ABSTRACT}

Aims: To analyze stress vulnerability in the work context and its effects on feeding.

Methods: Cross-sectional study of industrial workers from Santa Catarina state, southern Brazil. The occupational stress vulnerability scale (OSVS) and a structured questionnaire were used as instruments. Six sociodemographic and occupational variables (age, sex, schooling, work shift, work sector/unit, duration of employment), level of stress vulnerability (according to the OSVS), in addition to five nutritional variables which took into account self-reported stress rather than the vulnerability evaluated by the OSVS were assessed. Student's t test, Pearson's chi-square test, and linear trend were used for data analysis, and statistical significance was set at $5 \%(\mathrm{p} \leq 0.05)$.

Results: A total of 309 industrial workers were assessed. The mean age was $30.9 \pm 10.1$ years; $60.2 \%$ were women; $44.7 \%$ had attended high school; and $25.5 \%$ had finished elementary school. The median duration of employment was about 23 months (interquartile range of $7-57) ; 81.6 \%$ worked during the day, and $18.4 \%$ at night. The mean score of stress vulnerability was $22 \pm 11.6$ points; $249(80.6 \%)$ workers were classified at the lower levels of stress vulnerability and $60(19.4 \%)$ at the upper ones. There was association between increased stress vulnerability according to the OSVS and self-reported changes in feeding $(p=0.028)$. The prevalence of high stress vulnerability increased $(p=0.034)$ with duration of employment $(p=0.034)$. Under self-reported stress, women had more hunger, changes in feeding, and preference for sweets $(\mathrm{p}<0.001)$.

Conclusions: The industrial workers showed prevalence of lower levels of stress vulnerability, with pressure at work representing the main stressor. Higher levels of stress vulnerability were associated with increased duration of employment as well as with the perception that work causes stress and interferes in feeding. The finding that, under a self-reported occupational stress condition, the prevalence of perceived change in feeding was higher among women, associated with increased hunger and preference for sweets, indicates a greater vulnerability of this group, with potential risks for health problems.

KEY WORDS: feeding behavior; burnout, professional; occupational health; health vulnerability; stress, psychological. 
Abreviatura: EVENT, Escala de Vulnerabilidade ao Estresse no Trabalho.

\section{INTRODUÇÃO}

$\mathrm{Na}$ atualidade, as formas de adoecimento do trabalhador possuem relações com os diferentes tipos de gestão do trabalho e da produção. $\mathrm{O}$ mundo do trabalho vem se apresentando cada vez mais exigente e competitivo, impondo múltiplas demandas (físicas, cognitivas, psíquicas) que podem resultar em prejuízos na vida psicológica e social dos trabalhadores, chegando a níveis que podem caracterizar estresse ocupacional [1,2].

É importante considerar que o trabalho pode gerar ambivalência, ou seja, prazer-sofrimento. Segundo Linhart [3], se por um lado as pessoas não podem viver sem trabalho, o qual dá sentido e valor ao tempo livre e à vida, por outro lado está bastante presente a ideia de que o trabalho impede de aproveitar a vida, de ter tempo suficiente para viver [3]. Nesse sentido, o estresse relacionado ao trabalho pode ser entendido como um conjunto de situações que envolvem o ambiente de trabalho e que ameaçam as necessidades de realização pessoal e profissional ou a saúde, tanto física quanto mental [4].

Reconhece-se que o desgaste a que as pessoas estão expostas no âmbito do trabalho está, seguramente, implicado com processos de adoecimento [5]. Nesta direção, vêm sendo desenvolvidos estudos sobre vulnerabilidades no processo saúde-doença, em uma perspectiva que propõe identificar os fatores que condicionam a suscetibilidade das pessoas e dos coletivos, em seus contextos de vida, a agravos à saúde [6]. A vulnerabilidade constitui abordagem que inclui a noção da determinação social da saúde e da doença. Essa concepção compreende a saúde como processo de subjetivação estabelecida por contextos sociais, culturais e históricos específicos [7,8]. Dessa forma, segundo Ayres et al. [9], o conceito de vulnerabilidade pode ser entendido como um conjunto de aspectos individuais e coletivos relacionados à maior suscetibilidade de indivíduos e comunidades a um adoecimento ou agravo e, necessariamente, à menor disponibilidade de recursos para sua proteção [9].

Nesse cenário, e dada a centralidade que o trabalho vem assumindo na vida das pessoas [10], justifica-se o crescente interesse pelo estudo do estresse ocupacional e das possíveis variáveis a ele relacionadas, de forma a reconhecer os aspectos que vulnerabilizam a saúde dos indivíduos e dos grupos e aqueles que se constituem como recursos de proteção, passíveis de mobilização para o enfrentamento destas vulnerabilidades. Argumenta-se, portanto, pela relevância de que sejam realizados estudos que abordem diferentes aspectos implicados com a vulnerabilidade ao estresse ocupacional e que forneçam novos elementos para o desenvolvimento de programas de qualidade de vida no trabalho. Considerando que o estresse ocupacional pode alterar o apetite e a fome, resultando em prejuízos à saúde [11] e, por outro lado, que a alimentação pode ser orientada na direção de promover a saúde do trabalhador [12], sem perder de vista a perspectiva social, afetiva e cultural do ato de comer [13], desenvolveu-se este estudo, cujo objetivo foi analisar a vulnerabilidade ao estresse no contexto do trabalho e suas implicações com a alimentação.

\section{MÉTODOS}

Este foi um estudo transversal, cujos participantes foram trabalhadores de uma indústria de um município de pequeno porte, com taxa de urbanização de $77,45 \%$ e $62,64 \%$ de população economicamente ativa, situado na Mesorregião Oeste Catarinense [14]. A indústria, na época da pesquisa, contava com 353 trabalhadores, que foram esclarecidos e convidados para participar. Considerando-se aqueles que estavam em período de férias, em licença de saúde e os que se recusaram a participar da pesquisa, a população de estudo foi constituída por 309 trabalhadores, os quais assinaram termo de consentimento livre e esclarecido. Todos os procedimentos foram aprovados por Comitê de Ética em Pesquisa da Universidade Comunitária da Região de Chapecó (Unochapecó) sob Parecer n⿳丷ㅡ 039/2013, tendo sido rigorosamente respeitados todos os preceitos éticos vigentes da pesquisa envolvendo seres humanos.

A indústria onde o estudo foi realizado é do ramo alimentício, produtora de massas e biscoitos de diferentes tipos. É organizada em três unidades, sendo duas de produção e uma administrativa. As duas unidades de produção operam em turnos, que se iniciam às $6 \mathrm{~h}$ (Turno $\mathrm{A}$ ), às $10 \mathrm{~h} 40 \mathrm{~min}$ (Turno $\mathrm{B}$ ) e às $23 \mathrm{~h} 45 \mathrm{~min}$ (Turno $\mathrm{C}$ ), enquanto os setores administrativos operam em horário comercial (Turno D). As unidades de produção I e a administrativa estão localizadas em local próximo ao centro da cidade; já a unidade de produção II está situada em uma região mais distante do centro, e a empresa disponibiliza transporte coletivo próprio aos trabalhadores, o qual passa por vários pontos da cidade, em um deslocamento de cerca de 30 min. Destaca-se, de acordo com o escopo deste 
estudo, que a indústria não dispõe de restaurante/ refeitório ou espaço similar.

A coleta de dados iniciou-se com a aplicação da Escala de Vulnerabilidade ao Estresse no Trabalho (EVENT). Esse instrumento é uma escala psicométrica que avalia o quanto as circunstâncias do cotidiano do trabalho influenciam a conduta da pessoa, a ponto de caracterizar algum grau de vulnerabilidade ao estresse. A EVENT é composta por 40 questões relacionadas ao clima e funcionamento organizacional, à pressão no trabalho e à infraestrutura e rotina de trabalho [15]. Além destas questões, o instrumento contém um cabeçalho de identificação que contempla dados de idade, sexo, escolaridade e ocupação, tendo sido acrescentados, para esta pesquisa, espaços para informações sobre o setor e o tempo de trabalho na indústria.

$\mathrm{Na}$ sequência, foi aplicado aos participantes um questionário com cinco perguntas fechadas, de elaboração das pesquisadoras, a fim de identificar potenciais relações entre vulnerabilidade ao estresse (segundo a EVENT), estresse autorreferido e aspectos percebidos pelos participantes sobre sua alimentação. Ambos os instrumentos foram autoaplicados nos meses de agosto e setembro de 2013, em horário fora do expediente de trabalho dos participantes, estando uma das pesquisadoras presente e disponível para esclarecimento de dúvidas quanto ao seu preenchimento.

A partir dos dados coletados, foram analisadas seis variáveis sociodemográficas e ocupacionais (idade, sexo, escolaridade, turno de trabalho, setor/unidade de trabalho, tempo de trabalho), nível de vulnerabilidade ao estresse (segundo a EVENT) e, ainda, cinco variáveis alimentares, nas quais a expressão "sob condição de estresse" equivale a estresse autorreferido pelo participante do estudo e não ao nível de vulnerabilidade ao estresse avaliado por meio da EVENT. Essas variáveis de estresse autorreferido foram aferidas pelos seguintes itens presentes nos questionários: se a pessoa percebe alteração alimentar sob condição de estresse, tipo de alteração alimentar percebida sob condição de estresse, preferência alimentar sob condição de estresse, se o trabalho estressa, se o trabalho interfere na alimentação. A variável nível de vulnerabilidade ao estresse foi expressa em cinco níveis de estratificação, conforme escore gerado pela escala EVENT: nível inferior (até 24 pontos), nível médio inferior (25-35 pontos), nível médio (36 pontos), nível médiosuperior (37-45 pontos) e nível superior (igual ou superior a 46 pontos) [15].

A partir dos dados coletados, foi construído um banco de dados, utilizando-se o programa Excel $^{\circledR}$, o qual, uma vez finalizado, passou por análise de coerência e consistência. $\mathrm{Na}$ análise, as variáveis contínuas foram descritas por média e desvio padrão ou mediana e amplitude interquartílica, e as variáveis categóricas foram descritas por frequências absolutas e relativas. Para a comparação de médias entre os grupos, empregou-se o teste $t$ de Student. Na comparação de proporções entre os grupos foi utilizado o teste quiquadrado de Pearson. Para as variáveis ordinais, foi aplicado o teste qui-quadrado de tendência linear. $\mathrm{O}$ nível de significância adotado foi de $5 \%(\mathrm{p} \leq 0,05)$, e as análises foram realizadas no programa IBM SPSS Statistics versão 21.0.

\section{RESULTADOS}

A amostra foi composta por 309 trabalhadores $(87,5 \%$ de todos os trabalhadores da indústria pesquisada, na época do estudo). A média de idade observada foi de 30,9 $9 \pm 10,1$ anos, com mínimo de 17 e máximo de 63 anos. A caracterização completa da população em estudo está apresentada na Tabela 1.

Tabela 1. Caracterização sociodemográfica e ocupacional da amostra do estudo, constituída por 309 trabalhadores de uma indústria localizada em um município de pequeno porte da Mesorregião Oeste Catarinense, 2013.

\begin{tabular}{|c|c|}
\hline Variáveis & $N=309$ \\
\hline Idade (anos) - média \pm desvio padrão & $30,9 \pm 10,1$ \\
\hline \multicolumn{2}{|l|}{ Sexo - n (\%) } \\
\hline Masculino & $123(39,8)$ \\
\hline Feminino & $186(60,2)$ \\
\hline $\begin{array}{l}\text { Tempo na empresa (meses) - mediana (intervalo } \\
\text { interquartil) }\end{array}$ & $23(7-57)$ \\
\hline \multicolumn{2}{|l|}{ Turno - n (\%) } \\
\hline Turno A (início às 6:00h) & $97(31,4)$ \\
\hline Turno B (início às 10h:40min) & $98(31,7)$ \\
\hline Turno C (início às 23h:40min) & $57(18,4)$ \\
\hline Turno D (horário comercial) & $57(18,4)$ \\
\hline \multicolumn{2}{|l|}{ Nível de escolaridade - n (\%) } \\
\hline Fundamental incompleto & $50(16,2)$ \\
\hline Fundamental completo & $40(12,9)$ \\
\hline Médio incompleto & $39(12,6)$ \\
\hline Fundamental completo + médio incompleto & $79(25,5)$ \\
\hline Médio completo & $138(44,7)$ \\
\hline Superior incompleto & $18(5,8)$ \\
\hline Superior completo & $16(5,2)$ \\
\hline Especialização & $8(2,6)$ \\
\hline \multicolumn{2}{|l|}{ Unidade de Trabalho - n (\%) } \\
\hline Unidade de Produção & $291(94,2)$ \\
\hline Unidade Administrativa & $18(5,8)$ \\
\hline
\end{tabular}


Tabela 2. Fatores de vulnerabilidade ao estresse, segundo a Escala de Vulnerabilidade ao Estresse no trabalho (EVENT), em 309 trabalhadores de uma indústria localizada em um município de pequeno porte da Mesorregião Oeste Catarinense, 2013.

\begin{tabular}{l|c|c|c|cc|c}
\hline \multicolumn{1}{|c|}{ Fatores } & $\begin{array}{c}\text { Número de } \\
\text { itens }\end{array}$ & $\begin{array}{c}\text { Máximo da } \\
\text { pontuação no } \\
\text { instrumento }\end{array}$ & Média & Desvio padrão & Mediana & Min-Max \\
\hline Clima e Funcionamento Organizacional & 16 & 32 pontos & 8,07 & 5,80 & 7,00 & $0-28$ \\
\hline Pressão no Trabalho & 13 & 26 pontos & 9,76 & 4,96 & 10,00 & $0-24$ \\
\hline Infraestrutura e Rotina de Trabalho & 11 & 22 pontos & 4,28 & 2,96 & 4,00 & $0-17$ \\
\hline
\end{tabular}

A média do escore de vulnerabilidade ao estresse foi de 22 pontos $( \pm 11,6)$, com mínimo de zero e máximo de 56 pontos. A distribuição segundo os estratos da EVENT evidenciou que 249 (80,6\%) trabalhadores foram classificados nos níveis mais baixos de vulnerabilidade ao estresse: inferior 175 (56,6\%); médio-inferior $73(23,6 \%)$; e médio $1(0,3 \%)$; enquanto $60(19,4 \%)$ foram categorizados nos níveis mais elevados: médio-superior 51 (16,5\%); e superior $9(2,9 \%)$. Constatou-se que, isoladamente, o fator de maior peso no escore de vulnerabilidade ao estresse, conforme aferido pela EVENT, foi o da pressão no trabalho, cuja média constatada correspondeu a $37,5 \%$ da pontuação máxima possível de ser obtida pelo fator no instrumento (Tabela 2).

Tabela 3. Variáveis alimentares sob condição de estresse autorreferido no contexto do trabalho, em 309 trabalhadores de uma indústria localizada em um município de pequeno porte da Mesorregião Oeste Catarinense, 2013.

\begin{tabular}{|lr}
\hline \multicolumn{1}{|c|}{ Variáveis } & $\mathrm{n}(\%)$ \\
\hline Percebe alteração na alimentação sob estresse & $202(65,4)$ \\
Sim & $107(34,6)$ \\
Não & \\
\hline Tipo de alteração alimentar percebida sob estresse & $95(30,7)$ \\
$\quad$ Mais fome & $147(47,6)$ \\
Perde fome & $67(21,7)$ \\
Não muda a vontade de comer & \\
\hline Preferência alimentar sob estresse & $85(27,5)$ \\
\hline Doces & $32(10,4)$ \\
Salgados & $192(62,1)$ \\
Não tem preferência & \\
\hline O trabalho causa estresse & $94(30,4)$ \\
Raramente & $203(65,7)$ \\
Às vezes & $12(3,9)$ \\
Sempre & $129(41,7)$ \\
\hline O trabalho interfere na alimentação & $150(48,5)$ \\
Raramente & $30(9,7)$ \\
Às vezes &
\end{tabular}

Os dados sobre a alimentação no contexto do trabalho estão apresentados na Tabela 3, e evidenciam um perfil de trabalhadores que, predominantemente, considera que o trabalho interfere em sua alimentação, causando alterações sob condições de estresse. Essas alterações caracterizam-se, majoritariamente, como perda do apetite.

Quando foi testada a associação das variáveis em estudo com o nível de vulnerabilidade ao estresse dicotomizado (nível inferior/médio-inferior/médio vs nível médio-superior/superior), houve associação significativa com "alteração percebida na alimentação sob estresse" $(p=0,028)$. Trabalhadores que afirmaram perceber a ocorrência de alteração na alimentação quando estão sob condição de estresse apresentaram maior nível de vulnerabilidade ao estresse no trabalho quando comparados aos que disseram não perceber qualquer alteração em sua alimentação nestas condições $(23,3 \%$ vs $12,1 \%)$ (Tabela 4$)$.

Observou-se, também, que quanto mais trabalhadores afirmavam que "o trabalho causa estresse" e que "o trabalho interfere na alimentação", maior foi a prevalência de vulnerabilidade ao estresse em nível médio-superior/superior $(p=0,029$ e $p=0,001$ respectivamente) (Tabela 4). É importante ressaltar que a prevalência de vulnerabilidade ao estresse em nível médio-superior/superior aumentou significativamente com o aumento do tempo de trabalho na empresa, sendo de $16,7 \%$ para até dois anos e chegando a $31,6 \%$ quando este tempo superava os nove anos $(\mathrm{p}=0,034)$.

A prevalência de alteração percebida na alimentação sob condição de estresse foi significativamente maior entre as mulheres quando comparadas aos homens $(74,2 \%$ vs $52 \% ; \mathrm{p}<0,001)$. A variável sexo também esteve associada significativamente com a "preferência alimentar sob estresse": mulheres apresentaram uma maior preferência por comer doces quando comparadas aos homens $(37,1 \%$ vs $13,0 \%)$, enquanto uma maior proporção de trabalhadores homens, quando comparados às mulheres, referiu não ter preferência por alimentos doces ou salgados sob condição de estresse $(74,8 \%$ vs $53,8 \%)(\mathrm{p}<0,001)$. 
Tabela 4. Associação das variáveis em estudo com o nível de vulnerabilidade ao estresse em 309 industriários de um município de pequeno porte da Mesorregião Oeste Catarinense, 2013.

\begin{tabular}{|c|c|c|c|c|}
\hline \multirow{2}{*}{ Variáveis } & \multirow{2}{*}{$\mathbf{n}$} & \multicolumn{3}{|c|}{$\begin{array}{l}\text { Nível de vulnerabilidade ao estresse segundo a } \\
\text { Escala de Vulnerabilidade ao Estresse no Trabalho (EVENT) }\end{array}$} \\
\hline & & Inferior/Médio-Inferior/Médio & Médio-Superior/Superior & $\mathbf{p}$ \\
\hline Idade (anos) - média $\pm \mathrm{DP}$ & 309 & $30,8 \pm 10,0$ & $31,1 \pm 10,6$ & $0,879 *$ \\
\hline Sexo - n (\%) & & & & $0,484^{+}$ \\
\hline Masculino & 123 & $102(82,9)$ & $21(17,1)$ & \\
\hline Feminino & 186 & $147(79,0)$ & $39(21,0)$ & \\
\hline Turno - n (\%) & & & & $0,531^{+}$ \\
\hline 06:00-10:40 & 97 & $74(76,3)$ & $23(23,7)$ & \\
\hline $10: 40-14: 40$ & 98 & $83(84,7)$ & $15(15,3)$ & \\
\hline Comercial & 57 & $46(80,7)$ & $11(19,3)$ & \\
\hline Madrugada & 57 & $46(80,7)$ & $11(19,3)$ & \\
\hline Nível de escolaridade & & & & $0,447^{\ddagger}$ \\
\hline Fundamental incompleto & 50 & $40(80,0)$ & $10(20,0)$ & \\
\hline Fundamental completo & 40 & $33(82,5)$ & $7(17,5)$ & \\
\hline Médio incompleto & 39 & $37(94,9)$ & $2(5,1)$ & \\
\hline Médio completo & 138 & $106(76,8)$ & $32(23,2)$ & \\
\hline Superior incompleto & 18 & $15(83,3)$ & $3(16,7)$ & \\
\hline Superior completo/Especialização & 24 & $18(75,0)$ & $6(25,0)$ & \\
\hline Unidade de Trabalho & & & & $0,215^{+}$ \\
\hline Unidade de Produção & 291 & $232(79,7)$ & $59(20,3)$ & \\
\hline Unidade Administrativa & 18 & $17(94,4)$ & $1(5,6)$ & \\
\hline Percebe alteração na alimentação sob estresse & & & & $0,028^{+}$ \\
\hline Sim & 202 & $155(76,7)$ & $47(23,3)$ & \\
\hline Não & 107 & $94(87,9)$ & $13(12,1)$ & \\
\hline Tipo de alteração alimentar percebida sob estresse & & & & $0,438^{+}$ \\
\hline Tem mais fome & 95 & $79(83,2)$ & $16(16,8)$ & \\
\hline Perde fome & 147 & $114(77,6)$ & $33(22,4)$ & \\
\hline Não sente mudar a vontade de comer & 67 & $56(83,6)$ & $11(16,4)$ & \\
\hline Preferência alimentar sob estresse & & & & $0,447^{+}$ \\
\hline Doces & 85 & $70(82,4)$ & $15(17,6)$ & \\
\hline Salgados & 32 & $28(87,5)$ & $4(12,5)$ & \\
\hline Não tem preferência & 192 & $151(78,6)$ & $41(21,4)$ & \\
\hline O trabalho causa estresse & & & & $0,029^{+}$ \\
\hline Raramente & 94 & $82(87,2)$ & $12(12,8)$ & \\
\hline Às vezes & 203 & $159(78,3)$ & $44(21,7)$ & \\
\hline Sempre & 12 & $8(66,7)$ & $4(33,3)$ & \\
\hline O trabalho interfere na alimentação & & & & $0,001^{*}$ \\
\hline Raramente & 129 & $114(88,4)$ & $15(11,6)$ & \\
\hline Às vezes & 150 & $115(76,7)$ & $35(23,3)$ & \\
\hline Sempre & 30 & $20(66,7)$ & $10(33,3)$ & \\
\hline
\end{tabular}

* Teste t de Student; †Teste qui-quadrado de Pearson; ‡Teste qui-quadrado de tendência linear.

Além disso, quanto ao "tipo de alteração alimentar percebida sob estresse", as mulheres superaram a proporção de homens que afirmaram ter "mais fome" $(41,9 \%$ vs $13,8 \%$; $\mathrm{p}<0,001)$. Os homens, por sua vez, afirmaram mais que "não muda a vontade de comer" sob condição de estresse quando comparados às mulheres $(32,5 \%$ vs $14,5 \%$; $<<0,001)$.

Por outro lado, as associações da variável sexo com as variáveis "o trabalho causa estresse" $(p=0,094)$ e "o trabalho interfere na alimentação" ( $p=0,371)$ não 
foram significativas. Também não houve associação significativa entre turno de trabalho e "alteração percebida na alimentação sob estresse". As prevalências de alteração na alimentação foram semelhantes nos quatro turnos (66\% vs 64,3\% vs 66,7\% vs 64,9\%; $\mathrm{p}=0,990$ ). Por fim, não houve associação do turno de trabalho com as variáveis "o trabalho causa estresse" $(\mathrm{p}=0,762)$ e "o trabalho interfere na alimentação" $(\mathrm{p}=0,498)$.

\section{DISCUSSÃO}

A caracterização da população em estudo indica um perfil de participantes que são predominantemente jovens, mulheres, com ensino médio completo, pouco tempo de trabalho na empresa, atuando em período diurno em atividades dos setores da linha de produção. Este grupo apresentou baixo nível de vulnerabilidade ao estresse relacionado ao trabalho.

Nesse âmbito, cabe destacar que parece não haver um consenso na literatura quanto às relações entre nível de vulnerabilidade ao estresse e características como sexo, idade, escolaridade, turno e tempo de trabalho na empresa, com diferentes estudos apontando correlações positivas, negativas ou, ainda, ausência de qualquer correlação entre estas variáveis [16-19].

Neste estudo, entre as variáveis sociodemográficas e ocupacionais testadas, apenas tempo de trabalho na empresa esteve associada à vulnerabilidade ao estresse, tendo sido observado que a prevalência de vulnerabilidade em nível médio-superior/superior aumenta com o aumento do tempo de trabalho. Este achado pode ser explicado pelo fato de que o aumento do tempo de trabalho implica maior exposição às exigências desta atividade, o que repercute em menor capacidade de trabalho, que é a resultante do balanço entre as demandas do trabalho e os recursos de que o trabalhador dispõe para enfrentá-las, de forma a desempenhar satisfatoriamente sua atividade laboral [20].

A elevada prevalência de trabalhadores que apresentaram níveis de baixa vulnerabilidade ao estresse no trabalho (inferior/médio-inferior/médio) condiz com outros estudos que empregaram a mesma escala psicométrica, abordando porteiros, profissionais de saúde, universitários e trabalhadores em geral [16, 18,21-23]. Nesse sentido, Miguel e Noronha [17] afirmaram que, apesar de parecer não distinguir os níveis de vulnerabilidade ao estresse segundo variáveis como idade, sexo, escolaridade e outras, a EVENT tem se mostrado sensível para diferenciar trabalhadores que se encontram em níveis extremos do estresse.
Destaca-se que, dentre os fatores implicados na vulnerabilidade ao estresse de acordo com a EVENT, a pressão no trabalho foi o que apresentou maior pontuação no presente estudo, o que condiz com a literatura sobre o tema, que tem apontado a pressão como um dos eventos mais estressantes no trabalho [24-26]. Este fator expressa, em síntese, acúmulo de funções, de atividades e de responsabilidades [27], estando imbricado nos processos de precarização do trabalho [28].

Observou-se que, apesar da baixa vulnerabilidade ao estresse avaliada por meio da EVENT, a prevalência de estresse autorreferido relacionado ao trabalho ("o trabalho causa estresse") foi elevada, o que pode indicar uma compreensão sobre o que seja estresse, neste grupo, diferente daquela que a EVENT propõe medir.

Na mesma direção, alguns autores já destacaram que estresse é um construto que se tornou parte do senso comum, sendo o termo utilizado, por vezes, de forma indiscriminada, o que contribui para a distorção do seu significado [29,30]. Divergências significativas entre medidas psicométricas de vulnerabilidade ao estresse no trabalho e de estresse autorreferido podem indicar que estes dois construtos não sejam equivalentes [16], o que não significa que sejam utilizados inadequadamente em uma ou outra situação. Isso poderia, inclusive, justificar os achados aparentemente inconclusivos da literatura, conforme mencionado anteriormente, sobre as relações entre vulnerabilidade ao estresse e variáveis como idade, sexo, escolaridade, turno e tempo de trabalho, pelo menos em parte. Assim, considera-se que as divergências possam estar vinculadas, dependendo dos instrumentos empregados, à avaliação de construtos diferentes.

Além disso, há que se considerar que o estresse autorreferido possa estar mais ligado à ocorrência de fatores estressores eventuais no contexto do trabalho, mais ou menos negativos, do que propriamente a condições de caráter mais constante e que, portanto, seriam de mais difícil enfrentamento. Este argumento é suportado, neste estudo, pela observação de que a maioria dos participantes indicou que o trabalho causa estresse às vezes. Porém, ressalta-se que quanto mais trabalhadores afirmavam que o trabalho causa estresse, maior foi a prevalência de vulnerabilidade ao estresse no trabalho em nível médio-superior/ superior.

Da mesma forma, foi prevalente a afirmação de que o trabalho interfere na alimentação apenas ocasionalmente. Contudo, entre os que consideraram haver, em alguma medida, interferência do trabalho 
sobre a alimentação, maior foi a prevalência de vulnerabilidade ao estresse no trabalho em nível médio-superior/superior.

Seguindo a mesma dinâmica, foi observada associação entre alteração percebida na alimentação sob estresse autorreferido e um maior nível de vulnerabilidade ao estresse no trabalho, ou seja, os trabalhadores que afirmaram perceber alteração na alimentação sob estresse apresentaram maior nível de vulnerabilidade ao estresse no trabalho segundo a EVENT. Estes achados corroboram a afirmação de Nevanperä et al. [31], que enfatizaram a ocorrência de mudanças na alimentação dos trabalhadores em momentos de estresse. Em níveis mais exacerbados, também já foi relatado que há uma relação significativa entre nível alto de estresse no trabalho e o desenvolvimento de transtornos alimentares, como um mecanismo de enfrentamento para lidar com o estresse e os estados de humor negativos [32].

Portanto, com base nos achados apresentados, argumenta-se que há uma estreita relação entre as variáveis supracitadas, evidenciando maior suscetibilidade, ou ineficiência nas estratégias de enfrentamento para lidar com situações adversas do cotidiano de trabalho. Além disso, os resultados deste estudo condizem com os de estudo anterior, em que foi observado que o estresse ocupacional esteve relacionado à adoção de hábitos alimentares inadequados, principalmente no grupo dos mais estressados [33].

Cabe ressaltar as maiores prevalências observadas entre as mulheres participantes deste estudo para alteração percebida na alimentação sob estresse autorreferido, traduzindo-se predominantemente esta alteração por aumento da fome e preferência por alimentos doces. Estes achados convergem com os de estudo anterior, no âmbito desta temática, que indicou associação entre burnout, comportamento alimentar e ganho de peso entre mulheres trabalhadoras, enfatizando que em períodos estressantes ocorrem mudanças no comportamento alimentar que se caracterizam pela preferência por doces, refrigerantes e bebidas alcoólicas [31].

Importante também mencionar a indicação, na literatura, da existência de diferenças relevantes no modo de perceber (e referir) o estresse entre homens e mulheres [34], parecendo haver uma maior percepção negativa de estresse entre as mulheres. Esse aspecto se deve, provavelmente, à dupla jornada de trabalho, mais frequente entre as mulheres, ou ainda à falta de acesso e oportunidade às estratégias de controle do estresse [12].
Além disso, também tem sido apontado por alguns autores que as mulheres são mais predispostas ou vulneráveis a transtornos alimentares [35]. Neste cenário, cabe mencionar que o estereótipo de magreza e beleza pode contribuir para tornar as mulheres mais vulneráveis a agravos - de magnitudes diversas relacionados à alimentação, incluindo comportamentos de risco [36]. Considera-se que as implicações deste contexto podem produzir repercussões relevantes que extrapolam o nível individual, com potencial para atingir a saúde dos grupos sociais em que as mulheres trabalhadoras se inserem, na medida em que a mulher assume centralidade nos processos decisórios envolvidos com a alimentação da família [37].

Convém mencionar ainda que, nesta pesquisa, não foram observadas associações entre turno de trabalho e quaisquer outras variáveis testadas. Contudo, tem sido reconhecido que o trabalho em regime de turnos é contraditório em relação aos biorritmos humanos, contribuindo para processos de adoecimento diversos, especialmente no que se refere ao trabalho noturno [2,28]. Neste sentido, Arreal e López [38] salientaram, em estudo recente, que a alimentação é um direito universal e que, no âmbito da saúde do trabalhador, ainda está por receber maior atenção, principalmente em se tratando do trabalho noturno. Este achado e o fato de a maioria dos trabalhadores neste estudo apresentar níveis considerados baixos de vulnerabilidade ao estresse pode representar maior capacidade de resposta dos participantes frente às adversidades no trabalho. Os recursos que eles mobilizam no enfrentamento destas adversidades podem estar mais relacionados aos aspectos da dimensão individual da vulnerabilidade. A dimensão individual refere-se não somente aos aspectos biológicos, mas também à trajetória pessoal e psicossocial do indivíduo, envolvendo nível de conhecimento e escolaridade, valores, crenças, desejos, atitudes e discursos, assim como o grau e a qualidade das informações de que uma pessoa dispõe e o poder que tem para incorporá-las em suas práticas cotidianas [9]. Na perspectiva da teoria da vulnerabilidade, assume-se que algumas pessoas poderão estar menos vulneráveis do que outras diante de situações semelhantes, em decorrência dos recursos de que dispõem e de sua possibilidade de mobilizá-los efetivamente.

Assim, considerando o perfil dos participantes deste estudo, a baixa prevalência observada de vulnerabilidade ao estresse no trabalho pode estar relacionada a aspectos situados na dimensão individual, tais como a compatibilidade entre o nível de escolaridade e as exigências e expectativas em relação à ocupação dos trabalhadores, predominantemente em unidades de 
produção, como já sinalizado por Bachion et al. [16]. Além disso, o tempo de trabalho, relativamente baixo, e a prevalência de trabalho em período diurno também surgem como potenciais fatores de proteção à vulnerabilidade ao estresse $[11,20]$. Da mesma forma, no âmbito da dimensão social da vulnerabilidade, viver em municípios de pequeno porte, como neste estudo, parece oferecer mais recursos para o enfrentamento de situações estressoras [39].

Por outro lado, a análise no âmbito das variáveis alimentares relativas à condição de estresse autorreferido indica que as mulheres, neste grupo, estão mais vulneráveis ao estresse relacionado ao trabalho e a prejuízos daí decorrentes para a sua alimentação e saúde, conforme também já foi relatado por outros autores [32,35]. Nesse ponto, salienta-se que essa maior vulnerabilidade apresentada pelas mulheres pode estar situada no âmbito da dimensão social, que foca os aspectos contextuais que configuram vulnerabilidades individuais, considerando-se questões como liberdade de pensamento, relações raciais, étnicas e de gênero, entre outras [40]. Nesse sentido, importa registrar que o fato de as mulheres apresentarem características de maior vulnerabilidade, segundo as alterações alimentares sob condição de estresse autorreferido, tem o aspecto positivo de que, dada a sua maior percepção sobre estas questões, possam ser ampliadas suas possibilidades de construir e mobilizar recursos de enfrentamento.

Em síntese, este estudo constatou que níveis superiores de vulnerabilidade ao estresse estiveram associados ao aumento do tempo de trabalho na empresa, assim como à percepção de que o trabalho causa estresse e interfere na alimentação. No entanto, para o grupo de trabalhadores da indústria pesquisada, observou-se prevalência de níveis inferiores de vulnerabilidade ao estresse, o que pode ser explicado por uma suficiente capacidade de resposta às adversidades enfrentadas por eles no contexto do trabalho.

Argumenta-se que essa capacidade de resposta venha se constituindo, principalmente, a partir da dimensão individual, sugerindo-se que programas de promoção da qualidade de vida no trabalho sejam implantados, incluindo as condições de alimentação neste contexto, com especial atenção às mulheres trabalhadoras. Neste sentido, reforça-se que, sob condição de estresse autorreferido relacionado ao trabalho, a prevalência de alteração percebida na alimentação foi maior entre as mulheres, associada ao aumento da fome e à preferência por doces. Estes achados indicam uma maior suscetibilidade deste grupo na relação com o estresse autorreferido, com potenciais riscos de agravos à saúde.

Finalmente, considera-se relevante registrar que este estudo empreendeu uma aproximação em relação às associações entre vulnerabilidade ao estresse no trabalho e alimentação, levantando questões a serem aprofundadas e avaliadas sob diversas abordagens e perspectivas, de forma a adensar o conhecimento nesta interface ainda pouco explorada, contribuindo para a construção de recursos para o enfrentamento das vulnerabilidades à saúde do trabalhador.

\section{REFERÊNCIAS}

1. Fernandes RCP, Assunção AA, Carvalho FM. Mudanças nas formas de produção na indústria e a saúde dos trabalhadores. Ciênc Saúde Coletiva. 2010;15(Supl. 1):1563-74. http://dx.doi.org/10.1590/S1413-81232010000700068

2. Fonseca ISS, Araújo TM. Prevalência de transtornos mentais comuns em industriários da Bahia. Rev Bras Saúde Ocup. 2014;39(129): 35-49. http://dx.doi.org/10.1590/0303-7657000065012

3. Linhart D. A desmedida do capital. São Paulo: Boitempo; 2007. p. 42.

4. França ACL, Rodrigues LA. Stress, trabalho e doenças de adaptação. In: França ACL, Rodrigues LA. Stress e Trabalho: uma abordagem psicossomática. 4ํㅡㄹ. ed. São Paulo: Atlas; 2012. p. 29-55.

5. França ACL, Rodrigues LA. Uma perspectiva psicossocial em psicossomática via estresse e trabalho: In: Mello Filho J, Burd M. Psicossomática Hoje. 2ª ed. Porto Alegre: Artmed; 2010. p.111-34.

6. Nichiata LYI, Bertolozzi MR, Takahashi RF, Fracolli LA. A utilização do conceito de "vulnerabilidade" pela enfermagem. Rev Latino-Am Enfermagem. 2008;16(5):923-8. http://dx.doi.org/10.1590/S0104-11692008000500020

7. Sánchez AIM, Bertolozzi MR. Pode o conceito de vulnerabilidade apoiar a construção do conhecimento em Saúde Coletiva? Ciênc Saúde Coletiva. 2007;12(2):319-24. http://dx.doi.org/10.1590/S1413-81232007000200007

8. Gontijo DT, Medeiros M. Crianças e adolescentes em situação de rua: contribuições para compreensão dos processos de vulnerabilidade e desfiliação social. Ciênc Saúde Coletiva. 2009;14(2):467-75. http://dx.doi.org/10.1590/S1413-81232009000200015

9. Ayres JRCM, Paiva V, França Júnior, I. Conceitos e Práticas de prevenção: da história natural da doença ao quadro da vulnerabilidade e direitos humanos. In: Paiva V, Ayres JRCM, Buchalla CM, organizadores. Vulnerabilidade e direitos humanos: prevenção e promoção da saúde. Volume I: da doença à cidadania. Curitiba: Juruá; 2012. p. 71-94.

10. Aquino AS, Fernandes ACP. Qualidade de vida no trabalho. J Health Sci Institute. 2013;31(1):53-8. 
11. Franco JJD. Estrés alimentario y salud laboral vs. Estrés laboral y alimentacion equilibrada. Med Segur Trab. 2007;53(209):93-9.

12. Farah BQ, Barros MVG, Farias Júnior JC, Ritti-Dias RM, Lima RA, Barbosa JPAS, Nahas MV. Percepção de estresse: associação com a prática de atividades físicas no lazer e comportamentos sedentários em trabalhadores da indústria. Rev Bras Educ Fís Esporte 2013;27(2):225-34. http://dx.doi.org/10.1590/S1807-55092013000200007

13. Brasiliano S, Bucaretchi HÁ, Kachani AT. Aspectos psicológicos da alimentação. In: Cordás TA, Kachani AT, Lima AMS, Azevedo AP, organizadores. Nutrição em Psiquiatria. Porto Alegre: Artmed; 2010. p. 23-34.

14. Brasil. Instituto Brasileiro de Geografia e Estatística-IBGE. Censo Demográfico 2010 [internet]. Rio de Janeiro; 2010 [cited 2015 Apr 19 ]. Available from: http://www.cidades.ibge.gov.br/xtras/perfil.php?lang=\&codmun=421690\&search=santa-catarina|sao-lourenco-do-oeste

15. Sisto FF, Noronha APP, Santos, AAA, Batista MN. Normas. In: Sisto FF, Noronha APP, Santos, AAA, Batista MN. Escala de Vulnerabilidade ao Estresse no Trabalho: EVENT. São Paulo: Vetor, 2007.p. 73-74.

16. Bachion MM, Abreu LO, Godoy LF, Costa EC. Vulnerabilidade ao estresse entre professores de uma Universidade pública. Rev Enferm UERJ. 2005;13(1):32-7.

17. Miguel FK, Noronha APP. Estudo dos Parâmetros Psicométricos da Escala de Vulnerabilidade ao Estresse no Trabalho. Rev Evaluar. 2007;7:1-18.

18. Nunes COAT, Calais SL. Vulnerabilidade ao estresse no trabalho e percepção de suporte familiar em porteiros: um estudo correlacional. Psico-USF. 2011;16(1):57-65. http://dx.doi.org/10.1590/S1413-82712011000100007

19. Suehiro ACB, Santos AAA, Hatamoto CT, Cardoso MM. Vulnerabilidade ao estresse e satisfação no trabalho em profissionais do programa de saúde da família. Bol Psicol. 2008; 58(129):205-18

20. Martinez MC, Latorre MRD. O. Saúde e capacidade para o trabalho em trabalhadores de área administrativa. Rev Saúde Pública. 2006;40(5):851-8. http://dx.doi.org/10.1590/S0034-89102006000600015

21. Baptista MN, Carneiro AM. Validade da escala de depressão: relação com ansiedade e stress laboral. Estud Psicol. 2011;28(3):345-52. http://dx.doi.org/10.1590/s0103-166x2011000300006

22. Noronha APP, Fernandes DC. Estresse Laboral e Raciocínio Inferencial: Um Estudo Correlacional. Psicol Cienc Prof. 2007;27(4): 596-607. http://dx.doi.org/10.1590/S1414-98932007000400003

23. Oswaldo YC. Vulnerabilidade ao estresse no trabalho, Coping, depressão e qualidade de vida: Evidências de validade [tese]. [Itatiba]: Universidade de São Francisco; 2009. 145 p.

24. Baker D, Karasek RA. Stress. In: Levy BS, Wegman DH, editors. Occupational Health Recognizing and Preventing Work-Related Disease and Injury. 4thed. Philadelphia: Lippincott Williams \&Wilkins; 2000. p.419-36.

25. Glina DMR, Rocha LE, Batista ML, Mendonça MGV. Saúde Mental e Trabalho: uma reflexão sobre o nexo com o trabalho e o diagnóstico com base na prática. Cad Saúde Pública. 2001;17(3):607-16. http://dx.doi.org/10.1590/S0102-311X2001000300015

26. Schmidt DRC, Dantas RAS, Marziale MHP, Laus AM. Estresse ocupacional entre profissionais de enfermagem do bloco cirúrgico. Texto Contexto Enferm. 2009;18(2):330-7. http://dx.doi.org/10.1590/S0104-07072009000200017

27. Corrêa RZA, Souza MS, Baptista MN. Vulnerabilidade ao estresse no trabalho e qualidade de vida de enfermeiros. Psicol Argum. 2013;31(75):599-606. http://dx.doi.org/10.7213/psicol.argum.31.075.DS02

28. Franco T, Druck G, Seligmann-Silva E. As novas relações de trabalho, o desgaste mental do trabalhador e os transtornos mentais no trabalho precarizado. Rev Bras Saúde Ocup. 2010;35(122):229-48. http://dx.doi.org/10.1590/S0303-76572010000200006

29. Filgueiras JC, Hippert MIS. A polêmica em torno do conceito de estresse. Psicol Cienc Prof. 1999;19(3):40-51. http://dx.doi.org/10.1590/ S1414-98931999000300005

30. Oliveira ACF. Estresse. Brasília: Ministério da Educação; 2012.

31. Nevanperä NJ, Hopsu L, Kuosma E, Ukkola O, Uitti J, Laitinen J H. Occupational burnout, eating behavior, and weight among working women. Am J Clin Nutr. 2012;95(1):934-43. http://dx.doi.org/10.3945/ajcn.111.014191

32. King KA, Vidourek R, Schwiebert M. Disordered eating and job stress among nurses. J Nurs Manag. 2009;17(7):861-69. http://dx.doi. org/10.1111/j.1365-2834.2009.00969.x

33. Sociedad Espa-ola de Dietética y Ciencias de la Alimentación-SEDCA. El estrés en el trabajo incita al consumo de comida rápida [internet]. Madrid; 2012 [cited 2015 Apr 19]. Available from: http://www.webconsultas.com/dieta-y-nutricion/dieta-equilibrada/el-estres-en-eltrabajo-incita-al-consumo-de-comida-rapida-8701

34. Rossi AM. Homens X Mulheres: o Preço do Stress. Anais do IV Congresso de Stress; 2004 Jun 22-23; Porto Alegre: ISMA-BR; 2004.

35. Prisco APK, Araújo TM, Almeida MMG, Santos KOB. Prevalência de transtornos alimentares em trabalhadores urbanos de município do Nordeste do Brasil. Ciênc Saúde Coletiva. 2013;18(4):1109-18. http://dx.doi.org/10.1590/S1413-81232013000400024

36. Alvarenga MS, Scagliusi FB, Philippi ST. Comportamento de risco para transtorno alimentar em universitárias brasileiras. Rev Psiquiatr Clín. 2011;38(1):3-7.

37. Oliveira NRF, Vela HAG. Escolhas alimentares, decisões culturais: a mulher define o que vai pra mesa. Anais do $8^{\circ}$ Seminário Internacional Fazendo Gênero: Corpo, Violência e Poder; 2008 Ago 25-28; Florianópolis: UFSC; 2008.

38. Arreal JF, López LC. Trabalhadoras de turno noturno: relações de gênero, produção de vulnerabilidades e promoção da saúde. Rev Bras Saúde Ocup. 2014;39(130):184-97. http://dx.doi.org/10.1590/0303-7657000080113

39. Martins LF. Estresse ocupacional e esgotamento profissional entre profissionais da Atenção Primária à Saúde [dissertação]. [Juiz de Fora]: Universidade Federal de Juiz de Fora; 2011. 129 p.

40. Ayres JRCM, França Júnior I, Calazans GJ, Saletti Filho HC. O conceito de vulnerabilidade e as práticas de saúde: In: Czeresnia D, Freitas CM, organizadores. Promoção da saúde: conceitos, reflexões, tendências. Rio de Janeiro: Fiocruz; 2002. p. 117-39. 